\title{
Patient-Related Prognostic Factors for Anastomotic Leakage, Major Complications, and Short-Term Mortality Following Esophagectomy for Cancer: A Systematic Review and Meta-Analyses
}

\author{
Robert T. van Kooten, MD ${ }^{1}$ (1) , Daan M. Voeten, $\mathrm{MD}^{2}$, Ewout W. Steyerberg, PhD ${ }^{3}$, Henk H. Hartgrink, MD, PhD ${ }^{1}$, \\ Mark I. van Berge Henegouwen, MD, $\mathrm{PhD}^{2}$, Richard van Hillegersberg, MD, $\mathrm{PhD}^{4}$, Rob A. E. M. Tollenaar, MD, \\ $\mathbf{P h D}^{1}$, and Michel W. J. M. Wouters, MD, $\mathbf{P h D}^{3,5}$ \\ ${ }^{1}$ Department of Surgery, Leiden University Medical Center, Leiden, the Netherlands; ${ }^{2}$ Department of Surgery, Amsterdam \\ UMC, Cancer Center Amsterdam, University of Amsterdam, Amsterdam, the Netherlands; ${ }^{3}$ Department of Biomedical \\ Data Sciences, Leiden University Medical Center, Leiden, the Netherlands; ${ }^{4}$ Department of Surgery, University Medical \\ Center Utrecht, Utrecht, the Netherlands; ${ }^{5}$ Department of Surgery, Dutch Cancer Institute - Antoni van Leeuwenhoek \\ Hospital, Amsterdam, the Netherlands
}

\begin{abstract}
Objective. The aim of this study is to identify preoperative patient-related prognostic factors for anastomotic leakage, mortality, and major complications in patients undergoing oncological esophagectomy.

Background. Esophagectomy is a high-risk procedure with an incidence of major complications around $25 \%$ and short-term mortality around $4 \%$.

Methods. We systematically searched the Medline and Embase databases for studies investigating the associations between patient-related prognostic factors and anastomotic leakage, major postoperative complications (ClavienDindo $\geq$ IIIa), and/or 30-day/in-hospital mortality after esophagectomy for cancer.
\end{abstract}

ASA American Society of Anesthesiologists, CI Conference Interval, ERAS Enhanced Recovery After Surgery, OR Odds Ratio, QUIPS Quality in Prognostic Studies

Senior authors: Rob A. E. M. Tollenaar and Michel W. J. M. Wouters

(C) The Author(s) 2021

First Received: 14 June 2021

Accepted: 9 August 2021;

Published Online: 5 September 2021

R. T. van Kooten, MD

e-mail: r.t.van_kooten@lumc.nl
Results. Thirty-nine eligible studies identifying 37 prognostic factors were included. Cardiac comorbidity was associated with anastomotic leakage, major complications, and mortality. Male sex and diabetes were prognostic factors for anastomotic leakage and major complications. Additionally, American Society of Anesthesiologists (ASA) score $>$ III and renal disease were associated with anastomotic leakage and mortality. Pulmonary comorbidity, vascular comorbidity, hypertension, and adenocarcinoma tumor histology were identified as prognostic factors for anastomotic leakage. Age $>70$ years, habitual alcohol usage, and body mass index (BMI) $18.5-25 \mathrm{~kg} / \mathrm{m}^{2}$ were associated with increased risk for mortality.

Conclusions. Various patient-related prognostic factors are associated with anastomotic leakage, major postoperative complications, and postoperative mortality following oncological esophagectomy. This knowledge may define case-mix adjustment models used in benchmarking or auditing and may assist in selection of patients eligible for surgery or tailored perioperative care.

Esophageal carcinoma is the seventh most common and sixth most lethal malignancy worldwide. ${ }^{1}$ Its incidence is rising rapidly in the Western world, which might be a result of the obesity epidemic and the associated higher prevalence of gastroesophageal reflux disease. Currently, the 5-year survival rate of curatively treated esophageal 
carcinoma patients approximates $40-50 \%{ }^{2,3}$ This curative treatment consists of neoadjuvant chemo(radio)therapy followed by surgical resection. However, esophagectomy is a highly invasive procedure associated with significant postoperative morbidity. The incidence of major postoperative complications ranges around $26-31 \%$ with failureto-rescue rates of around $18-19 \%{ }^{4,5}$ Reduction of (severe) complications might reduce recovery time, length of hospital stay, readmission rates, and hospital costs, and increase long-term quality of life. In addition, recurrencefree and overall cancer-related survival are negatively affected by postoperative complications. ${ }^{6,7}$

The implementation of Enhanced Recovery After Surgery (ERAS) protocols reduces postoperative complication rates. ${ }^{8}$ Further reduction of major complications may be achieved by tailormade perioperative care using personalized prehabilitation programs. In addition, benchmarking surgical outcomes in national clinical audits might lead to a further decrease of surgical morbidity. ${ }^{9,10}$ An audit measures quality of care using structure, process, and outcome indicators and feeds benchmarked results back to clinicians. ${ }^{11,12}$ Reduction of hospital variation may enhance outcomes at population level. ${ }^{13}$ In auditing, knowledge on patient-related prognostic factors predicting adverse outcomes is essential to establish case-mix models enabling fair hospital comparison.

We aimed to identify patient-related prognostic factors for major postoperative complications (Clavien-Dindo $\geq$ IIIa), anastomotic leakage, and 30-day/in-hospital mortality after esophageal cancer surgery. ${ }^{14}$

\section{METHODS}

The study protocol was registered in the PROSPERO database (CRD42020204787). This systematic review and meta-analyses adhered to the Preferred Reporting Items for Systematic Reviews guidelines. The PRISMA checklist is provided in Supplementary File 1.

\section{Criteria for Study Eligibility}

All studies including patients undergoing curative-intent esophagectomy for cancer and describing patient-related prognostic factors for (1) anastomotic leakage, (2) major postoperative complications (Clavien-Dindo $\geq$ IIIa), and/ or (3) 30-day/in-hospital mortality were considered for inclusion. Studies including patients undergoing salvage or palliative surgery were excluded. No restrictions regarding neoadjuvant therapy or tumor stage were applied. Only retrospective or prospective cohort studies and randomized controlled trials with full-text articles published in English or Dutch were included. Case reports and case series $(<40$ patients) were excluded. Studies including children $(<18$ years of age) or animals were excluded. No restrictions as to study publication status were applied. In case of overlapping cohorts, the study reporting on the highest number of relevant outcome measures and/or patients was included.

\section{Search Method}

To identify all relevant publications, the Medline and Embase electronic databases were searched systematically from inception to 19 April 2021. Search terms included controlled MeSH terms in PubMed and EMtree terms in EMBASE, as well as free-text terms. The complete search strategy is presented in Supplementary File 2. No restrictions for date of publication were applied. Reference lists of identified review articles were checked for additional relevant studies. Authors were contacted in case of full-text unavailability.

\section{Study Selection}

Study selection was performed individually by D.M.V. and R.T.v.K. Initial screening was based on title and abstract. Disagreements regarding eligibility were resolved by discussion, with M.W.J.M.W. acting as arbitrator when necessary. Thereafter, full texts were independently screened by D.M.V. and R.T.v.K. Again, M.W.J.M.W. acted as arbitrator in case of disagreement. Reasons for exclusion were documented. A flowchart of study selection is depicted in Fig. 1. Endnote X9 (Clarivate Analytics, Philadelphia, PA) and Covidence were used during the selection process.

\section{Assessment of Risk of Bias}

All included studies were independently assessed for potential risk of bias by D.M.V. and R.T.v.K., using the Quality in Prognostic Studies (QUIPS) tool for classification of prognostic factor studies. ${ }^{15}$ Discrepancies were resolved by discussion, with M.W.J.M.W. as arbitrator. The risk of bias in studies was assessed in the following domains: study participation, study attrition, prognostic factor measurement, outcome measurement, adjustment bias, and statistical analysis bias. Each domain was graded as high, low, or unclear. The results are summarized in Supplementary File 3.

\section{Data Extraction and Management}

Data extraction was performed by R.T.v.K. and subsequently checked by D.M.V. The data extraction was 
FIG. 1. PRISMA flowchart of study selection

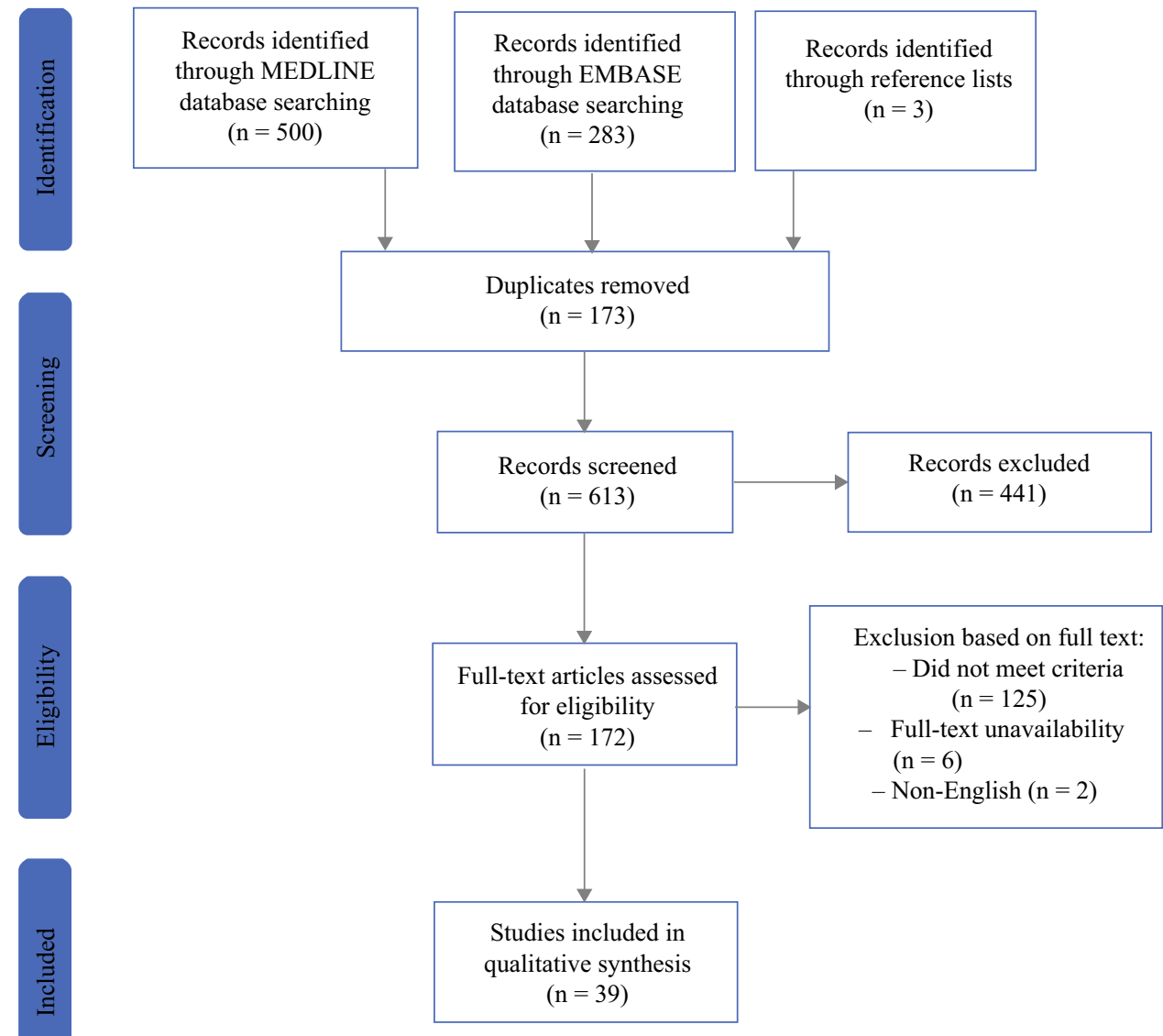

performed in a predefined Excel sheet, designed by D.M.V. and R.T.v.K. The sheet was piloted in at least one included study. Discrepancies regarding data extraction were resolved by discussion; M.W.J.M.W. acted as arbitrator when necessary. Subsequently, data were imputed in RevMan 5. The following data were extracted: (1) general study information (author, journal, year of publication, dataset, methodology, treatment regimen, and patient characteristics), (2) investigated patient-related prognostic factors, and (3) outcome measure incidence or odds ratios (OR) and accompanying 95\% confidence intervals (CI) of outcomes in different prognostic factor groups.

\section{Data Analyses}

Following data extraction, the homogeneity between the included studies was assessed using the Higgins $I^{2}$ statistic, with $I^{2}>50 \%$ considered heterogeneous. Random-effect modeling was used to calculate pooled univariable OR and accompanying 95\% CI for anastomotic leakage, major complications, and 30-day/in-hospital mortality. Two-sided $P<0.05$ was considered statistically significant. Analyses were conducted using RevMan 5 (Cochrane).

\section{RESULTS}

After removal of duplicates, the literature search resulted in a total of 613 studies potentially eligible for inclusion. After title and abstract, and full-text screening, 39 studies met inclusion criteria (Fig. 1). The risk of bias of the included studies is depicted in Supplementary File 3. All included studies were observational. The main study characteristics are presented in Table 1. A total of 37 different patient-related prognostic factors for severe complications, anastomotic leakage, and/or 30-day/in-hospital mortality were described in 48,853 patients and used in the current meta-analyses (Table 1). Eleven studies described prognostic factors for major complications, 31 for anastomotic leakage, and 12 for 30-day/in-hospital mortality.

\section{Anastomotic Leakage}

A total of 37 prognostic factors for anastomotic leakage were described in 31 studies; all were included in the metaanalyses (Table 2). Ten factors were significantly 
TABLE 1 Baseline characteristics of included studies

\begin{tabular}{|c|c|c|c|c|c|c|c|}
\hline Author (year) & Country & Study type & $\begin{array}{l}\text { Inclusion } \\
\text { period }\end{array}$ & $\begin{array}{l}\text { Number of } \\
\text { patients }\end{array}$ & Histology (ACC/SCC) & Localization & $\begin{array}{l}\text { Neoadjuvant } \\
\text { therapy }\end{array}$ \\
\hline Alexiou $^{16}$ & UK & Observational & 1987-1997 & 523 & $\mathrm{ACC}$ and SCC & $\begin{array}{l}\text { Cervical, intrathoracic, } \\
\text { and GEJ }\end{array}$ & $0 \%$ \\
\hline Aoyama $^{17}$ & Japan & Observational & 2005-2018 & 122 & $\mathrm{ACC}$ and $\mathrm{SCC}$ & Intrathoracic & - \\
\hline Berkelmans $^{18}$ & The Netherlands & Observational & 2013-2014 & 89 & $\mathrm{ACC}$ and $\mathrm{SCC}$ & - & $\begin{array}{l}\text { CRT } 73(82.0 \%) \\
\text { CTx } 4(4.5 \%)\end{array}$ \\
\hline Borggreve $^{19}$ & The Netherlands & Observational & 2003-2015 & 406 & $\begin{array}{l}\text { ACC } 309(76.1 \%), \text { SCC } 92 \\
\quad(22.7 \%)\end{array}$ & - & $\begin{array}{l}\text { CRT } 153(37.7 \%) \\
\text { CTx } 122(30.0 \%)\end{array}$ \\
\hline Busweiler $^{20}$ & $\begin{array}{l}\text { The Netherlands and } \\
\text { Sweden }\end{array}$ & Observational & 2012-2014 & 2.509 & $\begin{array}{l}\text { ACC } 1787(71.2 \%), \text { SCC } 415 \\
\quad(16.5 \%)\end{array}$ & $\begin{array}{l}\text { Cervical, intrathoracic } \\
\text { and GEJ }\end{array}$ & $\begin{array}{l}\text { CRT } 1857 \\
\quad(74.0 \%) \\
\text { CTx } 285(11.4 \%)\end{array}$ \\
\hline Daele $^{21}$ & Belgium & Observational & 2005-2014 & 412 & $\begin{array}{l}\text { ACC } 203(49.3 \%), \text { SCC } 209 \\
\quad(50.7 \%)\end{array}$ & Intrathoracic and GEJ & $\begin{array}{l}\text { RT } 195(47.3 \%) \\
\text { CTx } 228(55.3 \%)\end{array}$ \\
\hline Filip $^{22}$ & Italy & Observational & 2008-2012 & 167 & $\begin{array}{l}\text { ACC } 105(62.9 \%), \text { SCC } 58 \\
\quad(34.7 \%)\end{array}$ & $\begin{array}{l}\text { Cervical, intrathoracic } \\
\text { and GEJ }\end{array}$ & CRT $131(78.4 \%)$ \\
\hline Fjederholt $^{23}$ & Denmark & Observational & 2003-2012 & 557 & ACC $557(100.0 \%)$ & GEJ & - \\
\hline $\operatorname{Fogh}^{24}$ & USA & Observational & 1994-2005 & 260 & - & Intrathoracic and GEJ & CRT $260(100 \%)$ \\
\hline $\mathrm{Gao}^{25}$ & China & Observational & 2016-2017 & 96 & - & Intrathoracic and GEJ & $\begin{array}{l}\text { Unspecified } 38 \\
\quad(39.6 \%)\end{array}$ \\
\hline Goense $^{26}$ & The Netherlands & Observational & 2012-2015 & 167 & $\mathrm{ACC}$ and $\mathrm{SCC}$ & Intrathoracic and GEJ & $\begin{array}{l}\text { CRT } 8(4.8 \%) \\
\text { CTx } 145(86.8 \%)\end{array}$ \\
\hline Gooszen $^{27}$ & The Netherlands & Observational & 2011-2015 & 3.348 & $\begin{array}{l}\text { ACC } 2600(77.7 \%), \text { SCC } 663 \\
\quad(19.8 \%)\end{array}$ & Intrathoracic and GEJ & $\begin{array}{l}\text { CRT } 776(23.2 \%) \\
\text { CTx } 239(7.1 \%)\end{array}$ \\
\hline $\mathrm{Hall}^{28}$ & USA & Observational & 2005-2015 & 915 & $\begin{array}{l}\text { ACC } 682(74.5 \%), \text { SCC } 73 \\
\quad(8.0 \%)\end{array}$ & Intrathoracic and GEJ & $621(67.8 \%)$ \\
\hline Harustiak $^{29}$ & Czech Republic & Observational & 2005-2012 & 415 & $\mathrm{ACC}$ and $\mathrm{SCC}$ & - & $\begin{array}{l}\text { CRT } 93(22.4 \%) \\
\text { CTx } 139(33.5 \%)\end{array}$ \\
\hline Janowak $^{30}$ & USA & Observational & 2009-2013 & 168 & - & - & CRT 93 (55.4\%) \\
\hline Kassis $^{31}$ & USA & Observational & 2001-2011 & 7595 & - & - & $3478(45.8 \%)$ \\
\hline Kathiravetpillai $^{32}$ & The Netherlands & Observational & 2001-2014 & 190 & - & - & CRT $100 \%$ \\
\hline Klevebro $^{33}$ & Sweden & Observational & 2010-2017 & 2.332 & - & - & - \\
\hline Koeter $^{34}$ & The Netherlands & Observational & 2009-2011 & 53 & $\begin{array}{l}\mathrm{ACC} 49(92.5 \%), \mathrm{SCC} 4 \\
\quad(7.5 \%)\end{array}$ & - & CRT $100 \%$ \\
\hline Koyanagi $^{35}$ & Japan & Observational & 2014-2015 & 40 & $\begin{array}{l}\text { ACC } 4(10.0 \%), \text { SCC } 36 \\
\quad(90.0 \%)\end{array}$ & $\begin{array}{l}\text { Cervical, intrathoracic } \\
\text { and GEJ }\end{array}$ & $15(30.0 \%)$ \\
\hline Kruhilikava $^{36}$ & Denmark & Observational & 2003-2010 & 285 & - & - & - \\
\hline Markar $^{37}$ & USA & Observational & 1991-2011 & 500 & - & - & - \\
\hline $\mathrm{McBee}^{38}$ & USA & Observational & 2016-2018 & 1.260 & $\mathrm{ACC}$ and $\mathrm{SCC}$ & $\begin{array}{l}\text { Cervical, intrathoracic } \\
\text { and GEJ }\end{array}$ & - \\
\hline $\mathrm{Miki}^{39}$ & Japan & Observational & 2000-2015 & 158 & - & $\begin{array}{l}\text { Cervical, intrathoracic } \\
\text { and GEJ }\end{array}$ & CTx $35(22.2 \%)$ \\
\hline Mitzman $^{40}$ & USA & Observational & 2009-2016 & 9.389 & - & - & - \\
\hline Miyawaki $^{41}$ & Japan & Observational & 2013-2017 & 188 & $\mathrm{ACC}$ and SCC & - & - \\
\hline Murphy $^{42}$ & USA & Observational & 2002-2008 & 191 & - & - & - \\
\hline Okamura $^{43}$ & Japan & Observational & 2011-2015 & 300 & ACC and SCC & - & - \\
\hline Rutegard $^{44}$ & Sweden & Observational & 2001-2005 & 567 & $\begin{array}{l}\text { ACC } 466(82.2 \%), \text { SCC } 149 \\
\quad(26.7 \%)\end{array}$ & $\begin{array}{l}\text { Cervical, intrathoracic } \\
\text { and GEJ }\end{array}$ & $33(5.8 \%)$ \\
\hline Rutegard $^{45}$ & Sweden & Observational & 2001-2005 & 559 & $\begin{array}{l}\text { ACC } 449(80.3 \%), \text { SCC } 110 \\
\quad(19.7 \%)\end{array}$ & $\begin{array}{l}\text { Cervical, intrathoracic } \\
\text { and GEJ }\end{array}$ & $29(5.2 \%)$ \\
\hline Saito $^{46}$ & Japan & Observational & 2007-2015 & 90 & $\begin{array}{l}\text { ACC } 3(3.3 \%), \text { SCC } 87 \\
\quad(96.7 \%)\end{array}$ & - & CTx $29(32.2 \%)$ \\
\hline Salem $^{47}$ & USA & Observational & 2010-2013 & 129 & - & - & - \\
\hline Sato $^{48}$ & Japan & Observational & 2013-2019 & 248 & $\begin{array}{l}\text { ACC } 213(85.9 \%), \text { SCC } 21 \\
\quad(8.5 \%)\end{array}$ & - & - \\
\hline
\end{tabular}


Table 1 (continued)

\begin{tabular}{|c|c|c|c|c|c|c|c|c|c|c|c|}
\hline Author (year) & Country & & \multicolumn{2}{|c|}{ Study type } & $\begin{array}{l}\text { Inclusion } \\
\text { period }\end{array}$ & $\begin{array}{l}\text { Number of } \\
\text { patients }\end{array}$ & \multicolumn{2}{|c|}{ Histology (ACC/SCC) } & \multicolumn{2}{|l|}{ Localization } & $\begin{array}{l}\text { Neoadjuvant } \\
\text { therapy }\end{array}$ \\
\hline Scarpa $^{49}$ & Italy & & \multicolumn{2}{|c|}{ Observational } & 2008-2012 & 181 & \multicolumn{2}{|l|}{-} & \multicolumn{2}{|l|}{-} & - \\
\hline Schlottmann ${ }^{50}$ & USA & & \multicolumn{2}{|c|}{ Observational } & $2000-2014$ & 5.243 & \multicolumn{2}{|l|}{-} & \multicolumn{2}{|l|}{-} & - \\
\hline Shichinohe ${ }^{51}$ & Japan & & \multicolumn{2}{|c|}{ Observational } & 2009-2012 & 483 & \multicolumn{2}{|l|}{-} & \multicolumn{2}{|l|}{-} & - \\
\hline Takeuchi $^{52}$ & Japan & & \multicolumn{2}{|c|}{ Observational } & 2011 & 5.354 & \multicolumn{2}{|c|}{$\mathrm{ACC}$ and $\mathrm{SCC}$} & \multicolumn{2}{|c|}{$\begin{array}{l}\text { Cervical, intrathoracic } \\
\text { and GEJ }\end{array}$} & $1.005(18.8 \%)$ \\
\hline Werf $^{53}$ & The Netherland & & \multicolumn{2}{|c|}{ Observational } & 2011-2016 & 3.091 & \multicolumn{2}{|c|}{$\mathrm{ACC}$ and $\mathrm{SCC}$} & \multicolumn{2}{|c|}{ Intrathoracic and GEJ } & $\begin{array}{l}\text { CRT } 3.091 \\
\quad(100 \%)\end{array}$ \\
\hline Zhao $^{54}$ & China & & \multicolumn{2}{|c|}{ Observational } & 2010-2016 & 273 & \multicolumn{2}{|c|}{ SCC $273(100.0 \%)$} & \multicolumn{2}{|l|}{-} & $0 \%$ \\
\hline Author (year) & MI/open/both & \multicolumn{2}{|c|}{$\begin{array}{l}\text { Transthoracic/ } \\
\text { transhiatal }\end{array}$} & \multicolumn{2}{|c|}{$\begin{array}{l}\text { Location of } \\
\text { anastomosis }\end{array}$} & $\begin{array}{l}\text { Type of } \\
\text { anastomosis }\end{array}$ & $\mathrm{AL}$ & $\begin{array}{l}\text { Major } \\
\text { complications* }\end{array}$ & $\begin{array}{l}\text { 30-Day } \\
\text { mortality }\end{array}$ & \multicolumn{2}{|c|}{$\begin{array}{l}\text { Investigated } \\
\text { prognostic factors }\end{array}$} \\
\hline Alexiou $^{16}$ & $100 \%$ open & \multicolumn{2}{|l|}{-} & \multicolumn{2}{|c|}{ Thoracic } & - & $29(5.5 \%)$ & - & - & \multicolumn{2}{|l|}{ Age } \\
\hline Aoyama $^{17}$ & - & \multicolumn{2}{|l|}{-} & \multicolumn{2}{|c|}{ Cervical } & - & $44(36.1 \%)$ & - & - & \multicolumn{2}{|c|}{$\begin{array}{l}\text { Age, sex, smoking, alcohol } \\
\text { usage, tumor stage }\end{array}$} \\
\hline Berkelmans $^{18}$ & $100 \% \mathrm{MI}$ & Both & & - & & - & $15(16.9 \%)$ & - & - & $\begin{array}{r}\text { Sex, ne } \\
\text { ASA } \\
\text { com } \\
\text { card } \\
\text { com } \\
\text { com } \\
\text { com } \\
\text { rena } \\
\text { BM }\end{array}$ & $\begin{array}{l}\text { djuvant therapy, } \\
\text { core, any } \\
\text { bidity, } \\
\text { vascular } \\
\text { bidity, pulmonary } \\
\text { bidity, vascular } \\
\text { bidity, diabetes, } \\
\text { lisease, steroid use, }\end{array}$ \\
\hline Borggreve $^{19}$ & Both & Both & & - & & $\begin{array}{l}\text { Handsewn and } \\
\text { stapled }\end{array}$ & $104(25.6 \%)$ & - & - & $\begin{array}{l}\text { Sex, hi } \\
\text { ther } \\
\text { card } \\
\text { com } \\
\text { com } \\
\text { smo }\end{array}$ & $\begin{array}{l}\text { logy, neoadjuvant } \\
\text { y, ASA score, } \\
\text { vascular } \\
\text { bidity, pulmonary } \\
\text { bidity, diabetes, } \\
\text { g }\end{array}$ \\
\hline Busweiler $^{20}$ & Both & Both & & $\begin{array}{r}\text { Cervi } \\
\text { tho }\end{array}$ & $\begin{array}{l}\text { al and } \\
\text { acic }\end{array}$ & - & $311(12.4 \%)$ & - & $59(2.4 \%)$ & Age, se & ASA score \\
\hline Daele $^{21}$ & Both & Trans & toracic & Thora & & Stapled & $12(3.0 \%)$ & - & - & $\begin{array}{r}\text { Age, se } \\
\text { neos } \\
\text { neoz } \\
\text { cher } \\
\text { card } \\
\text { com } \\
\text { rena } \\
\text { hype } \\
\text { surg } \\
\text { preo }\end{array}$ & $\begin{array}{l}\text { histology, } \\
\text { uvant radiotherapy, } \\
\text { uvant } \\
\text { therapy, ASA score, } \\
\text { vascular } \\
\text { bidity, diabetes, } \\
\text { lisease, } \\
\text { ension, previous } \\
\text {, smoking, } \\
\text { rative weight loss }\end{array}$ \\
\hline Filip $^{22}$ & Both & - & & - & & - & - & $20(12.0 \%)$ & - & $\begin{array}{l}\text { Sex, tu } \\
\text { hist } \\
\text { ther } \\
\text { card } \\
\text { com } \\
\text { com } \\
\text { com } \\
\text { rena } \\
\text { dise } \\
\text { weis }\end{array}$ & $\begin{array}{l}\text { gy localization, } \\
\text { gy, neoadjuvant } \\
\text { y, ASA score, } \\
\text { vascular } \\
\text { bidity, pulmonary } \\
\text { bidity, vascular } \\
\text { bidity, diabetes, } \\
\text { lisease, hepatic } \\
\text {, HIV, preoperative } \\
\text { loss }\end{array}$ \\
\hline Fjederholt $^{23}$ & - & Both & & - & & - & $42(7.5 \%)$ & - & - & $\begin{array}{r}\text { Sex, A } \\
\text { inde } \\
\text { stag }\end{array}$ & $\begin{array}{l}\text { score, Charlson } \\
\text { smoking, tumor }\end{array}$ \\
\hline $\mathrm{Fogh}^{24}$ & - & - & & - & & - & $32(12.3 \%)$ & - & $14(5.4 \%)$ & Age, se & \\
\hline $\mathrm{Gao}^{25}$ & $100 \% \mathrm{MI}$ & Both & & - & & $\begin{array}{l}\text { Handsewn and } \\
\text { stapled }\end{array}$ & $12(12.5 \%)$ & - & - & $\begin{array}{r}\text { Age, se } \\
\text { ther } \\
\text { puln } \\
\text { diab } \\
\text { alco }\end{array}$ & $\begin{array}{l}\text { neoadjuvant } \\
\text {, comorbidity, } \\
\text { nary comorbidity, } \\
\text { es, hypertension, } \\
\text { l }\end{array}$ \\
\hline
\end{tabular}


Table 1 (continued)

\begin{tabular}{|c|c|c|c|c|c|c|c|c|}
\hline Author (year) & MI/open/both & $\begin{array}{l}\text { Transthoracic/ } \\
\text { transhiatal }\end{array}$ & $\begin{array}{l}\text { Location of } \\
\text { anastomosis }\end{array}$ & $\begin{array}{l}\text { Type of } \\
\text { anastomosis }\end{array}$ & $\mathrm{AL}$ & $\begin{array}{l}\text { Major } \\
\text { complications* }\end{array}$ & $\begin{array}{l}\text { 30-Day } \\
\text { mortality }\end{array}$ & $\begin{array}{l}\text { Investigated } \\
\text { prognostic factors }\end{array}$ \\
\hline Goense $^{26}$ & $100 \% \mathrm{MI}$ & Both & - & $\begin{array}{l}\text { Handsewn and } \\
\text { stapled }\end{array}$ & $40(24.0 \%)$ & - & - & $\begin{array}{l}\text { Sex, neoadjuvant therapy, } \\
\text { ASA score, cardiovascular } \\
\text { comorbidity, pulmonary } \\
\text { comorbidity, vascular } \\
\text { comorbidity, diabetes, } \\
\text { renal disease, } \\
\text { hypertension, smoking }\end{array}$ \\
\hline Gooszen $^{27}$ & Both & - & $\begin{array}{c}\text { Cervical and } \\
\text { thoracic }\end{array}$ & & $656(19.6 \%)$ & - & - & $\begin{array}{l}\text { Sex, tumor localization, } \\
\text { histology, neoadjuvant } \\
\text { therapy, ASA score, } \\
\text { cardiovascular } \\
\text { comorbidity, pulmonary } \\
\text { comorbidity, vascular } \\
\text { comorbidity, diabetes, } \\
\text { neurological comorbidity, } \\
\text { hypertension, previous } \\
\text { surgery, tumor stage }\end{array}$ \\
\hline Hall $^{28}$ & Both & - & - & - & $127(13.9 \%)$ & - & - & $\begin{array}{l}\text { Sex, histology, neoadjuvant } \\
\text { radiotherapy, ASA score, } \\
\text { ADL dependency, } \\
\text { cardiovascular } \\
\text { comorbidity, pulmonary } \\
\text { comorbidity, bleeding } \\
\text { disorder, diabetes, renal } \\
\text { disease, steroid use, } \\
\text { smoking, preoperative } \\
\text { weight loss, tumor stage }\end{array}$ \\
\hline Harustiak $^{29}$ & - & Both & Thoracic & $\begin{array}{l}\text { Handsewn and } \\
\text { stapled }\end{array}$ & $56(13.5 \%)$ & - & - & $\begin{array}{l}\text { Sex, neoadjuvant therapy, } \\
\text { neoadjuvant therapy, } \\
\text { diabetes, hypertension, } \\
\text { BMI }\end{array}$ \\
\hline Janowak $^{30}$ & Both & Both & - & - & - & $58(35.0 \%)$ & - & $\begin{array}{l}\text { Age, sex, neoadjuvant } \\
\text { therapy, ASA score, } \\
\text { cardiovascular } \\
\text { comorbidity, pulmonary } \\
\text { comorbidity, diabetes, } \\
\text { renal disease, smoking, } \\
\text { BMI }\end{array}$ \\
\hline Kassis $^{31}$ & Both & Both & - & - & $804(10.6 \%)$ & - & - & $\begin{array}{l}\text { Sex, neoadjuvant therapy, } \\
\text { ASA score, cardiovascular } \\
\text { comorbidity, vascular } \\
\text { comorbidity, diabetes, } \\
\text { renal disease, previous } \\
\text { surgery, hypertension, } \\
\text { steroid use, history of } \\
\text { malignancy, smoking, } \\
\text { BMI }\end{array}$ \\
\hline Kathiravetpillai ${ }^{32}$ & Both & - & - & - & $50(26.3 \%)$ & $39(20.5 \%)$ & $9(4.7 \%)$ & $\begin{array}{l}\text { Interval neoadjuvant and } \\
\text { surgery }\end{array}$ \\
\hline Klevebro $^{33}$ & Both & - & - & - & $312(13.3 \%)$ & $1383(59.3 \%)$ & $42(1.8 \%)$ & $\begin{array}{l}\text { Cardiovascular comorbidity, } \\
\text { pulmonary comorbidity }\end{array}$ \\
\hline Koeter $^{34}$ & Both & - & Cervical & $\begin{array}{l}\text { Handsewn and } \\
\text { stapled }\end{array}$ & $13(24.5 \%)$ & - & - & $\begin{array}{l}\text { Sex, histology, ASA score, } \\
\text { comorbidity }\end{array}$ \\
\hline Koyanagi $^{35}$ & Both & Both & Cervical & $\begin{array}{l}\text { Handsewn and } \\
\text { stapled }\end{array}$ & $7(17.5 \%)$ & - & - & $\begin{array}{l}\text { Sex, tumor localization, } \\
\text { histology, neoadjuvant } \\
\text { therapy, smoking, tumor } \\
\text { stage }\end{array}$ \\
\hline Kruhilikava $^{36}$ & - & - & - & - & $24(8.4 \%)$ & $62(21.8 \%)$ & $7(2.5 \%)$ & BMI \\
\hline Markar $^{37}$ & - & - & - & - & $18(3.6 \%)$ & - & $3(0.6 \%)$ & Age \\
\hline $\mathrm{McBee}^{38}$ & Both & - & - & - & $171(13.6 \%)$ & - & $34(2.7 \%)$ & $\mathrm{BMI} \geq 30 \mathrm{~kg} / \mathrm{m}^{2}$ \\
\hline
\end{tabular}


Table 1 (continued)

\begin{tabular}{|c|c|c|c|c|c|c|c|c|}
\hline Author (year) & MI/open/both & $\begin{array}{l}\text { Transthoracic/ } \\
\text { transhiatal }\end{array}$ & $\begin{array}{l}\text { Location of } \\
\text { anastomosis }\end{array}$ & $\begin{array}{l}\text { Type of } \\
\text { anastomosis }\end{array}$ & $\mathrm{AL}$ & $\begin{array}{l}\text { Major } \\
\text { complications* }\end{array}$ & $\begin{array}{l}\text { 30-Day } \\
\text { mortality }\end{array}$ & $\begin{array}{l}\text { Investigated } \\
\text { prognostic factors }\end{array}$ \\
\hline $\mathrm{Miki}^{39}$ & $100 \% \mathrm{MI}$ & - & - & - & - & $30(23.4 \%)$ & - & $\begin{array}{l}\text { Age, sex, tumor localization, } \\
\text { neoadjuvant therapy, } \\
\text { pulmonary comorbidity, } \\
\text { diabetes, BMI }<25 \mathrm{~kg} / \mathrm{m}^{2}\end{array}$ \\
\hline Mitzman $^{40}$ & Both & - & - & - & - & - & $321(3.4 \%)$ & BMI \\
\hline Miyawaki ${ }^{41}$ & - & Transthoracic & Cervical & Handsewn & $29(15.4 \%)$ & - & - & $\begin{array}{l}\text { Sex, neoadjuvant therapy } \\
\text { pulmonary comorbidity, } \\
\text { diabetes, hypertension, } \\
\text { tumor stage }\end{array}$ \\
\hline Murphy $^{42}$ & Both & - & - & - & $16(8.4 \%)$ & - & - & $\begin{array}{l}\text { Comorbidity, smoking, } \\
\text { alcohol, tumor stage }\end{array}$ \\
\hline Okamura $^{43}$ & Both & - & Cervical & $\begin{array}{l}\text { Handsewn and } \\
\text { stapled }\end{array}$ & $35(11.7 \%)$ & - & - & $\begin{array}{l}\text { Age, sex, histology, } \\
\text { neoadjuvant therapy, } \\
\text { cardiovascular } \\
\text { comorbidity, pulmonary } \\
\text { comorbidity, diabetes, } \\
\text { neurological comorbidity, } \\
\text { hepatic disease, } \\
\text { hypertension, smoking, } \\
\text { HbA1c }\end{array}$ \\
\hline Rutegard $^{44}$ & - & Both & Thoracic & - & - & $154(25.0 \%)$ & - & $\begin{array}{l}\text { Sex, histology, neoadjuvant } \\
\text { therapy, any comorbidity, } \\
\text { tumor stage }\end{array}$ \\
\hline Rutegard $^{45}$ & - & Both & Thoracic & $\begin{array}{l}\text { Handsewn and } \\
\text { stapled }\end{array}$ & $44(7.9 \%)$ & - & - & $\begin{array}{l}\text { Sex, histology, neoadjuvant } \\
\text { therapy, any comorbidity, } \\
\text { tumor stage }\end{array}$ \\
\hline Saito $^{46}$ & $100 \% \mathrm{MI}$ & Both & - & - & - & $32(35.6 \%)$ & - & $\begin{array}{l}\text { Sex, histology, neoadjuvant } \\
\text { therapy, ASA score, } \\
\text { cardiovascular } \\
\text { comorbidity, diabetes, } \\
\text { smoking, alcohol, BMI, } \\
\text { tumor stage }\end{array}$ \\
\hline Salem $^{47}$ & $100 \% \mathrm{MI}$ & - & - & - & $5(3.9 \%)$ & - & - & BMI \\
\hline Sato $^{48}$ & Both & - & Thoracic & - & $38(15.3 \%)$ & - & - & $\begin{array}{l}\text { Sex, histology, neoadjuvant } \\
\text { therapy, pulmonary } \\
\text { comorbidity, diabetes, } \\
\text { hypertension, tumor stage }\end{array}$ \\
\hline Scarpa $^{49}$ & Both & Both & - & - & $8(4.4 \%)$ & $20(11.0 \%)$ & $2(1.1 \%)$ & Age \\
\hline Schlottmann ${ }^{50}$ & - & - & - & - & $297(5.7 \%)$ & - & - & Age \\
\hline Shichinohe ${ }^{51}$ & - & - & - & - & $54(11.1 \%)$ & $132(27.3 \%)$ & - & Sex, malnutrition \\
\hline Takeuchi $^{52}$ & Both & Both & Both & - & - & - & $244(4.6 \%)$ & $\begin{array}{l}\text { Sex, neoadjuvant therapy, } \\
\text { ASA score, renal disease }\end{array}$ \\
\hline Werf $^{53}$ & Both & Both & $\begin{array}{l}\text { Cervical and } \\
\text { thoracic }\end{array}$ & $\begin{array}{l}\text { Hand sewn and } \\
\text { stapled }\end{array}$ & $341(11.0 \%)$ & $185(6.0 \%)$ & $106(3.4 \%)$ & $\begin{array}{l}\text { Interval neoadjuvant and } \\
\text { surgery }\end{array}$ \\
\hline Zhao $^{54}$ & $100 \% \mathrm{MI}$ & - & - & & $19(7.0 \%)$ & $25(9.2 \%)$ & $0(0.0 \%)$ & Age \\
\hline
\end{tabular}

$A S A$ American Society of Anesthesiologists, $A L$ anastomotic leakage, $B M I$ body mass index, $G E J$ gastroesophageal junction, $M I$ minimally invasive, $A C C$ adenocarcinoma, $C R T$ chemoradiotherapy, $C T x$ chemotherapy, $R T$ radiotherapy, $S C C$ squamous cell carcinoma

*Major complications defined as Clavien-Dindo $\geq$ IIIa 
TABLE 2 Results of meta-analyses identifying patient-related prognostic factors for anastomotic leakage

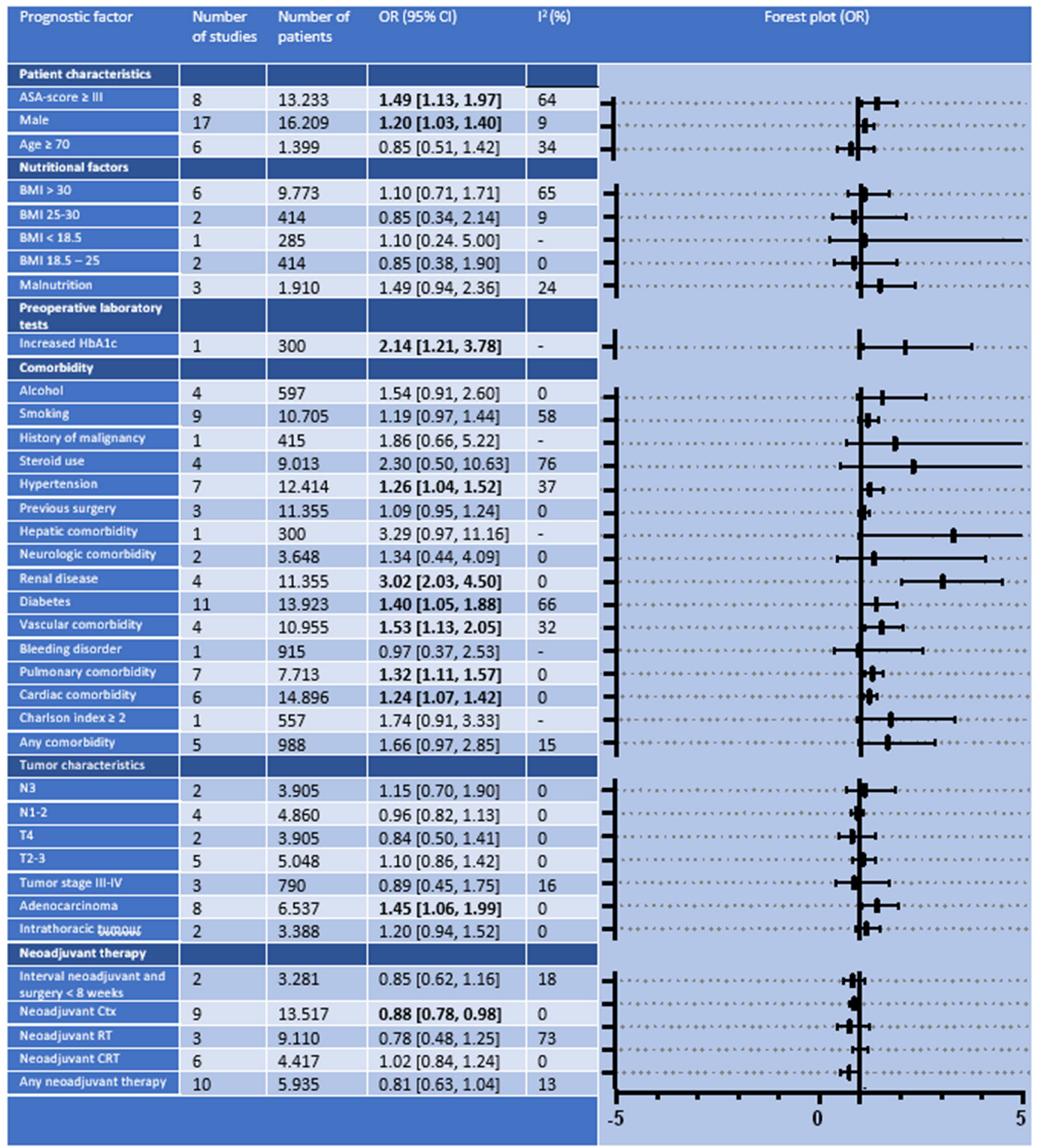

ASA American Society of Anesthesiologists, BMI body mass index, CRT chemoradiotherapy, Ctx chemotherapy, OR odds ratio, RT radiotherapy 
associated with anastomotic leakage, and one protective factor was identified.

Renal disease was the most prominent prognostic factor for anastomotic leakage with an OR of 3.02 (95\% CI $2.03-4.50 ; P<0.01)$. In addition, vascular comorbidity (OR 1.53; 95\% CI 1.13-2.05; $P<0.01$ ), diabetes (OR 1.40 ; $95 \%$ CI $1.05-1.88 ; P<0.01)$, pulmonary comorbidity (OR $1.32 ; 95 \%$ CI $1.11-1.57 ; \quad P<0.01$ ), hypertension (OR 1.26; 95\% CI 1.04-1.52; $P=0.02$ ), and cardiac comorbidity (OR 1.24; 95\% CI 1.07-1.42; $P<0.01)$ were significantly associated with anastomotic leakage. ASA score $\geq$ III also significantly increased the risk of anastomotic leakage (OR 1.49; 95\% CI 1.13-1.97; $P=0.04)$. Males were at greater risk for anastomotic leakage than females (OR 1.20; 95\% CI 1.03-1.40; $P=0.02$ ). Anastomotic leakage occurred more often after surgery for adenocarcinoma compared with squamous cell carcinoma (OR 1.45; 95\% CI 1.06-1.99; $P=0.02$ ).

Increased hemoglobin $\mathrm{A} 1 \mathrm{c}(\mathrm{HbA} 1 \mathrm{c})$ was also associated with anastomotic leakage (OR 2.14; 95\% CI 1.21-3.78; $P<0.01)$ but was only described by one study. ${ }^{43}$ Therefore, meta-analysis was not possible.

Patients receiving neoadjuvant chemotherapy were at lower risk for anastomotic leakage (OR 0.88; 95\% CI $0.78-0.98 ; P=0.04)$.

An analysis of studies only including minimally invasive esophagectomy showed no significant associations (Supplementary File 4).

\section{Major Complications}

A total of 23 prognostic factors for major postoperative complications $(\mathrm{CD} \geq \mathrm{IIIa})$ were described in 11 studies and were used in the meta-analyses (Table 3). Of these factors, four were significantly associated with major complications, of which male sex was the most prominent (OR 4.50; 95\% CI 1.21-16.64; $P=0.02$ ). In addition, cardiac comorbidity (OR 1.53; 95\% CI 1.25-1.87; $P<0.01$ ) and diabetes (OR 1.93; 95\% CI 1.14-3.26; $P=0.01)$ were significantly associated with major complications. The presence of any comorbidity was also associated with major complications but was described in only one study (OR $1.69 ; 95 \%$ CI $1.12-2.55 ; P=0.01)$. A time interval between neoadjuvant therapy and surgery of $<8$ weeks was associated with fewer major complications (OR 0.68; 95\% CI $0.50-0.93 ; P=0.01$ ).

\section{Mortality}

Fifteen prognostic factors for 30-day/in-hospital mortality were identified in 12 studies and used for metaanalyses (Table 4). Of these factors, six were significantly associated with increased mortality rates. ASA $\geq$ III (OR
2.77; 95\% CI 1.80-4.26; $P<0.01$ ), cardiac comorbidity (OR 2.40; 95\% CI 1.72-3.35; $P<0.01$ ), age 70 years or older (OR 2.06; 95\% CI 1.66-2.56; $P<0.01$ ), and BMI of $18.5-25 \mathrm{~kg} / \mathrm{m}^{2}$ (OR 1.41; 95\% CI $\left.1.11-1.78 ; P<0.01\right)$ were significantly associated with higher risk of mortality. In addition, habitual alcohol usage (OR 3.10; 95\% CI $2.26-4.25 ; P<0.01)$ and renal disease (OR 2.85; 95\% CI $1.71-4.74 ; P<0.01)$ were significantly associated with increased mortality rates but were described in only one study. Overweight (BMI $\left.25-30 \mathrm{~kg} / \mathrm{m}^{2}\right)(\mathrm{OR} 0.40 ; 95 \% \mathrm{CI}$ $0.30-0.53 ; P<0.01)$ and an interval between neoadjuvant therapy and surgery of $<8$ weeks (OR $0.54 ; 95 \%$ CI $0.35-0.85 ; P<0.01)$ were associated with lower mortality rates.

\section{DISCUSSION}

This is the first study to present a systematic review and meta-analyses describing prognostic factors for anastomotic leakage, major complications, and 30-day/in-hospital mortality following esophageal cancer surgery. Thirty-nine studies were included, providing a comprehensive and quantitative overview of the available literature. After analyses of 37 potential prognostic factors described in literature, renal disease, vascular comorbidity, diabetes, pulmonary, hypertension, cardiac comorbidity, ASA score $\geq$ III, male sex, and adenocarcinoma tumor histology were prognostic factors for anastomotic leakage. Patients receiving neoadjuvant chemotherapy had a lower risk for anastomotic leakage. Male sex, cardiac comorbidity, and diabetes were prognostic factors for major complications. In the current study, age $>70$ years, ASA score $\geq$ III, cardiac comorbidity, and BMI of $18.5-20 \mathrm{~kg} /$ $\mathrm{m}^{2}$ were prognostic factors for mortality whereas BMI of $25-30 \mathrm{~kg} / \mathrm{m}^{2}$ appeared preventive of mortality. A time interval of $<8$ weeks between neoadjuvant therapy and surgery was associated with lower major complication and mortality rates.

\section{Patient Characteristics}

Although the observed associations were heterogeneous, this study shows that male sex was associated with both higher anastomotic leakage and major complication rates. This might be a result of the higher incidence of smoking and alcohol consumption in the male population. ${ }^{55}$ Another theory described in literature is that cortisol-induced sex hormones vary among men and women, making males more susceptible to postoperative complications after surgically induced stress. ${ }^{56}$ In the current study, older patients are at higher risk for postoperative mortality than younger patients; age did not seem to impact anastomotic leakage 
TABLE 3 Results of meta-analyses identifying patient-related prognostic factors for major complications (Clavien-Dindo $\geq$ IIIa)

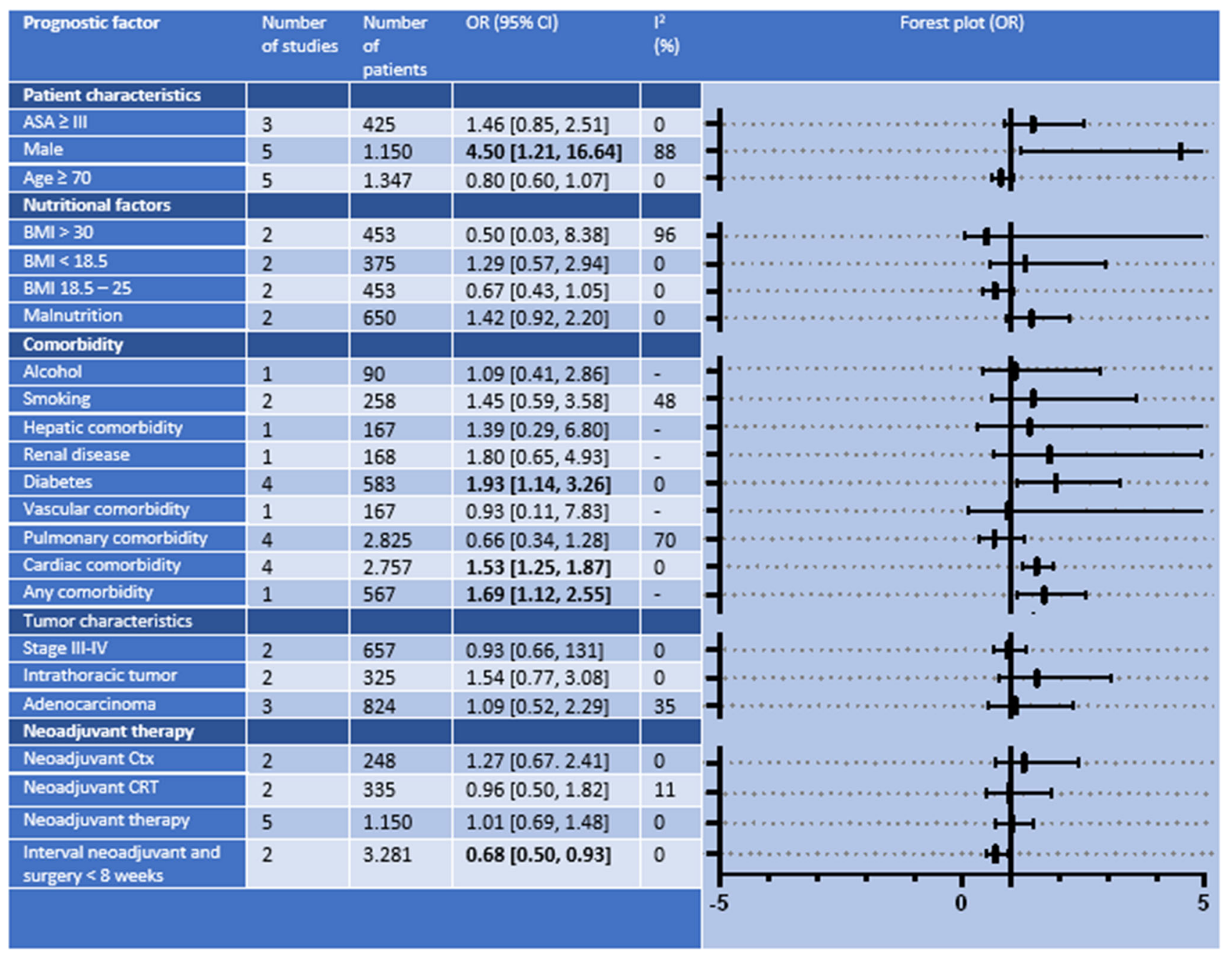

ASA American Society of Anesthesiologists, BMI body mass index, Ctx chemotherapy, $C R T$ chemoradiotherapy, $O R$ odds ratio

and major complication rates. This might be caused by an increased susceptibility for failure to rescue in the elderly. ${ }^{5}$ In the elderly, decreased preoperative performance status as demonstrated by a higher ASA score and/or comorbidities, such as cardiac and pulmonary comorbidity, might result in worse short-term outcomes. ${ }^{33}$

\section{Comorbidity}

As shown in this study, comorbidity is a prognostic factor for the occurrence of postoperative complications. The presence of comorbidities might, besides poorer physical performance, also implicate a greater presence of artery calcifications, which was shown by Goense et al. to be independently associated with anastomotic leakage. ${ }^{57}$ Additionally, the association between diabetes and major complications is well understood, as hyperglycemia induces microvascular damage that subsequently reduces healing capacity. ${ }^{58}$

\section{Body Mass Index}

This study showed that BMI of $18.5-20 \mathrm{~kg} / \mathrm{m}^{2}$ is associated with postoperative mortality. Patients with BMI between 25 and $30 \mathrm{~kg} / \mathrm{m}^{2}$, however, tended to have lower risk for mortality. Previous studies have shown that preoperative weight loss and a lower BMI make patients more susceptible for failure to rescue. ${ }^{4,5}$ Patients with higher 
TABLE 4 Results of meta-analyses identifying patient-related prognostic factors for 30-day/in-hospital mortality

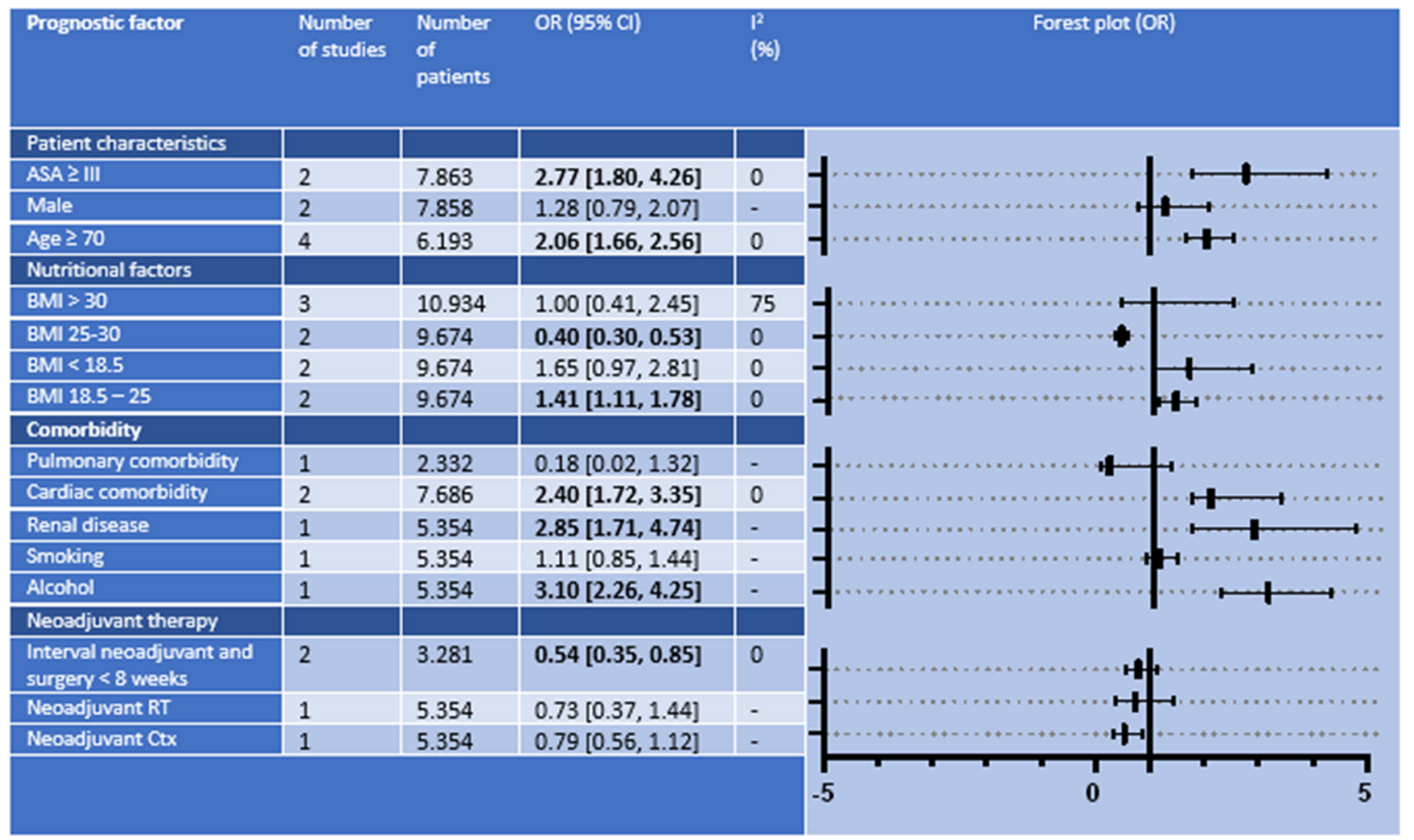

ASA American Society of Anesthesiologists, BMI body mass index, $C t x$ chemotherapy, $O R$ odds ratio, $R T$ radiotherapy

BMI at baseline might have more physical reserves (i.e., be less prone for catabolism), which prevents short-term adverse events. An even higher BMI (>30 kg/m ${ }^{2}$ ) was not protective for mortality. This might be caused by the difficulty of surgery in the obese due to the high amount of visceral fat compromising intraoperative visibility and making the surgery more challenging. ${ }^{59}$

\section{Neoadjuvant Therapy}

The current study also shows lower leakage rates after administration of neoadjuvant therapy. As the administration of neoadjuvant therapy is the standard of care for esophageal cancer, it may only be omitted in frail patients unable to withstand systemic therapy. This might explain the lower anastomotic leakage rates in patients undergoing neoadjuvant therapy compared with patients not receiving preoperative systemic therapy. Another prognostic factor observed in this study was the interval between neoadjuvant therapy and surgery. This study shows that an interval of $>8$ weeks is associated with increased major complication and mortality rates. The higher rate of adverse events in patients with a prolonged interval may be subjected to selection bias. Van de Werf et al. showed that more frail patients had a longer interval. ${ }^{53}$ In these frail patients, the interval might have been used for preoperative optimization. Another explanation may be that the interval is prolonged due to toxicity and/or slower recovery from neoadjuvant therapy. However, especially in patients undergoing chemoradiotherapy, the longer interval might also complicate surgery because of increased postradiation scarring with increasing interval lengths.

\section{Tumor Histology}

As shown in this study, adenocarcinoma tumor histology is a prognostic factor for anastomotic leakage after esophagectomy. A theory is that, based on the differences in pathogenesis of adenocarcinoma and squamous carcinoma, patient characteristics are different. For instance, adenocarcinoma is more common in overweight and obese patients, and in patients with more alcohol usage both are risk factors for anastomotic leakage. ${ }^{36}$ However, squamous cell carcinoma is more common in patients with habitual 
alcohol usage and smoking. ${ }^{60}$ Another difference between adenocarcinoma and squamous cell carcinoma is the localization, since adenocarcinoma is typically located more proximally. This localization is more suitable for cervical anastomosis, which is associated with a higher frequency of anastomotic leakage. ${ }^{27}$

\section{Surgical Techniques}

Given the differences in incidence and severity of anastomotic leakage of cervical versus intrathoracic anastomosis, the risk factors for anastomotic leakage might also differ based on anastomotic location. ${ }^{27}$ Additionally, minimally invasive surgery is being used more in daily practice, but unfortunately many studies do not report open and minimally invasive procedures separately. Therefore, this meta-analysis was unable to make distinctions between different surgical techniques (e.g., location of anastomosis, minimally invasive surgery), since the included studies did not allow for stratified analyses.

\section{Perioperative Care}

The identification of prognostic factors for adverse events after esophagectomy may provide opportunities to optimize perioperative care by treating or optimizing these prognostic factors preoperatively and thereby decreasing surgical risk. Reduction of postoperative morbidity and mortality may in turn reduce healthcare costs. ${ }^{61}$ Therefore, reduction of postoperative morbidity impacts healthcare at patient, hospital, and national levels. The prognostic factors described in the current study may contribute to focused and personalized preoperative care by enrolling patients with certain prognostic factors into (tailormade) prehabilitation programs. Currently, more generalized perioperative care programs are being studied and implemented in the form of ERAS protocols. ${ }^{62}$ As part of the ERAS protocols, lifestyle interventions (e.g., alcohol cessation) are introduced in daily practice. ${ }^{63-65}$ In addition, there is more focus on preoperative malnutrition and impaired physical capacity, which are shown to be negative prognostic factors for postoperative complications in this meta-analysis. ${ }^{66}$ Intra- and postoperative care are also being standardized in ERAS protocols (e.g., fluid therapy, opioid-sparing analgesia). ${ }^{67}$

The reduction of postoperative complications is important because complications are associated with reduced overall survival. Additionally, the reduction of complications positively impacts (progression-free) survival. ${ }^{68}$ It is thought that infectious complications lead to release of proinflammatory cytokines, which are related to tumor progression and metastasis. ${ }^{69}$ One might even argue that resection could be reconsidered in patients with multiple prognostic factors as definitive chemoradiotherapy might be a more well suited curative treatment option for such patients. $^{70,71}$ However, one should keep in mind the reduced survival after definitive chemoradiotherapy.

With the use of neoadjuvant therapy, a window for preoperative optimization is opened. A systematic review showed that (p)rehabilitation programs for esophageal cancer patients can improve objective measures of physical fitness. However, effects on postoperative outcomes were less eminent. ${ }^{72}$ Nonetheless, preoperative exercise programs have been shown to significantly impact healthrelated quality of life. ${ }^{73}$ Several studies report that welldesigned randomized controlled trials on prehabilitation programs are needed in order to prove their beneficial effects on short-term postoperative outcomes. ${ }^{72,74}$ They should focus on optimizable preoperative prognostic factors (e.g., malnutrition or vitamin deficiencies). Esophageal cancer patients are at high risk for malnutrition due to the anatomical localization of the tumor. Therefore, nutritional interventions are important in preoperative prehabilitation. ${ }^{75}$ This is supported by the results of the current study showing that patients with low BMI have increased risk of postoperative mortality. Slightly overweight patients even had reduced mortality rates. These results indicate that malnourishment and depletion of essential food substances are an important and modifiable prognostic factor in esophageal cancer surgery.

Identification of high-risk patients may indicate that changes in postoperative care are needed, for example, closer postoperative surveillance or delayed enteral feeding in high-risk patients. Closer postoperative surveillance might for instance be done by using wearable devices for continuous postoperative monitoring, even on the regular hospital ward. This might lead to more timely recognition and identification of postoperative adverse events, subsequently leading to earlier treatment and lower failure-torescue rates. $^{76,77}$

The identified prognostic factors for major adverse outcomes after surgery are vital in clinical auditing. Comparing hospitals and providing clinicians with benchmarked outcome information is an important quality improvement tool. ${ }^{78}$ For fair hospital comparison, benchmarked information should be corrected for differences in case mix among hospitals. The current study provides prognostic factors for three major adverse events after esophagectomy that should be used for case-mix correction in clinical audits such as the Dutch Upper Gastrointestinal Cancer Audit (DUCA). ${ }^{79}$

\section{Limitations}

This study had some limitations. Firstly, it provided an overview of multiple studies, creating a heterogeneous 
patient population. Additionally, definitions of prognostic factors (e.g., renal disease, cardiac comorbidity) used in literature are heterogeneous, making interpretation difficult. In addition, neoadjuvant therapy is currently standard of care, but this is not yet incorporated in all studies, compromising the external validity of the current study. As discussed, ERAS protocols influence postoperative outcomes, which may interfere with the results of this metaanalysis. However, none of the included studies reported on the use of ERAS protocols. The observational study design used in all the included studies may have hindered adequate interpretation of results. Additionally, most of the included studies were retrospective. Therefore, the current study is subjected to bias. However, it is suspected that, due to the high number of studies and patients included, this bias was limited. In current prognostic factor research, several limitations are known, such as publication bias, reporting bias, poor statistical analysis, and inadequate replication of findings. ${ }^{80}$ These meta-analyses used pooled data to calculate univariable ORs, which do not correct for potential confounding factors. Additionally, this study focuses on preoperative prognostic factors, whereas surgical factors, such as the type of anastomosis or surgery, may also contribute to the risk of postoperative major complications. Lastly, continuous variables such as BMI and age are reported as categorical variables, which is subjected to bias and may make risk estimates less useful. ${ }^{81}$

Future research should be directed towards prospective studies with well-documented prognostic factors, in addition to well-designed randomized controlled trials investigating the impact of preoperative prehabilitation programs for modifiable prognostic factors on surgical outcomes and quality of life. This should pave the way to enhanced personalized perioperative care.

\section{Conclusion}

In conclusion, this systematic review and meta-analyses identified 37 prognostic factors that are associated with adverse events after esophageal cancer surgery. Cardiac comorbidity was identified as a prognostic factor for all three studied adverse outcomes (anastomotic leakage, major complications, and mortality). Male sex and diabetes were identified as prognostic factors for anastomotic leakage and major complications. ASA score > III and renal disease were shown to be associated with anastomotic leakage and mortality. Pulmonary comorbidity, vascular comorbidity, hypertension, and adenocarcinoma were prognostic factors for anastomotic leakage. Older age $(>70$ years), habitual alcohol usage, and intermediate BMI were associated with increased risk for mortality. These factors should be used in case-mix correction models in national clinical audits. In addition, they also enable further research for accurate preoperative patient selection and personalized perioperative care ultimately aiming to reduce surgical morbidity and improve postoperative quality of life.

Supplementary Information The online version contains supplementary material available at https://doi.org/10.1245/s10434021-10734-3.

ACKNOWLEGDEMENT This study was supported by the Leiden University Medical Center (LUMC), Leiden, the Netherlands.

DISCLOSURES M.I.v.B.H. is consultant for Mylan, Johnson \& Johnson, Alesi Surgical, and Medtronic, and received research grants from Olympus and Stryker. R.v.H. is consultant for Medtronic and proctoring surgeon for Intuitive Surgical Inc. and trains other surgeons in robot-assisted minimally invasive esophagectomy. For the remaining authors no conflicts of interest were declared.

OPEN ACCESS This article is licensed under a Creative Commons Attribution 4.0 International License, which permits use, sharing, adaptation, distribution and reproduction in any medium or format, as long as you give appropriate credit to the original author(s) and the source, provide a link to the Creative Commons licence, and indicate if changes were made. The images or other third party material in this article are included in the article's Creative Commons licence, unless indicated otherwise in a credit line to the material. If material is not included in the article's Creative Commons licence and your intended use is not permitted by statutory regulation or exceeds the permitted use, you will need to obtain permission directly from the copyright holder. To view a copy of this licence, visit http://creativecommons. org/licenses/by/4.0/.

\section{REFERENCES}

1. Bray F, Ferlay J, Soerjomataram I, Siegel RL, Torre LA, Jemal A. Global cancer statistics 2018: GLOBOCAN estimates of incidence and mortality worldwide for 36 cancers in 185 countries. CA Cancer J Clin. 2018;68(6):394-424.

2. Ruol A, Castoro C, Portale G, et al. Trends in management and prognosis for esophageal cancer surgery: twenty-five years of experience at a single institution. Arch Surg. 2009;144(3):247-54.

3. van Hagen P, Hulshof MC, van Lanschot JJ, et al. Preoperative chemoradiotherapy for esophageal or junctional cancer. $N$ Engl $J$ Med. 2012;366(22):2074-84.

4. Busweiler LA, Henneman D, Dikken JL, et al. Failure-to-rescue in patients undergoing surgery for esophageal or gastric cancer. Eur J Surg Oncol. 2017;43(10):1962-9.

5. Abdelsattar ZM, Habermann E, Borah BJ, Moriarty JP, Rojas RL, Blackmon SH. Understanding failure to rescue after esophagectomy in the United States. Ann Thorac Surg. 2020;109(3):865-71.

6. Voeten DM, van der Werf LR, van Sandick JW, van Hillegersberg R, van Berge Henegouwen MI. Length of hospital stay after uncomplicated esophagectomy. Hospital variation shows room for nationwide improvement. Surg Endosc. 2020.

7. Aurello P, Berardi G, Moschetta G, et al. Recurrence following anastomotic leakage after surgery for carcinoma of the distal esophagus and gastroesophageal junction: a systematic review. Anticancer Res. 2019;39(4):1651-60. 
8. Ashok A, Niyogi D, Ranganathan P, et al. The enhanced recovery after surgery (ERAS) protocol to promote recovery following esophageal cancer resection. Surg Today. 2020.

9. Wouters MW, Jansen-Landheer ML, van de Velde CJ. The Quality of Cancer Care initiative in the Netherlands. Eur J Surg Oncol. 2010;36(Suppl 1):S3-s13.

10. Marang-van de Mheen PJ, Dijs-Elsinga J, Otten W, et al. The relative importance of quality of care information when choosing a hospital for surgical treatment: a hospital choice experiment. Med Decis Making. 2011;31(6):816-27.

11. Waljee J, McGlinn EP, Sears ED, Chung KC. Patient expectations and patient-reported outcomes in surgery: a systematic review. Surgery. 2014;155(5):799-808.

12. Dimick JB, Welch HG, Birkmeyer JD. Surgical mortality as an indicator of hospital quality: the problem with small sample size. JAMA. 2004;292(7):847-51.

13. Cleary PD, McNeil BJ. Patient satisfaction as an indicator of quality care. Inquiry. 1988;25(1):25-36.

14. Clavien PA, Barkun J, de Oliveira ML, et al. The Clavien-Dindo classification of surgical complications: five-year experience. Ann Surg. 2009;250(2):187-96.

15. Hayden JA, van der Windt DA, Cartwright JL, Côté P, Bombardier C. Assessing bias in studies of prognostic factors. Ann Intern Med. 2013;158(4):280-6.

16. Alexiou C, Beggs D, Salama FD, Brackenbury ET, Morgan WE. Surgery for esophageal cancer in elderly patients: the view from Nottingham. J Thorac Cadiovasc Surg. 1998;116(4):545-53.

17. Aoyama T, Atsumi Y, Hara K, Tamagawa H, et al. Risk factors for postoperative anastomosis leak after esophagectomy for esophageal cancer. Vivo. 2020;34(2):857-62.

18. Berkelmans GH, Kouwenhoven EA, Smeets BJ, et al. Diagnostic value of drain amylase for detecting intrathoracic leakage after esophagectomy. World J Gastroenterol. 2015;21(30):9118-25.

19. Borggreve AS, Goense L, van Rossum PSN, van Hillegersberg R, de Jong PA, Ruurda JP. Generalized cardiovascular disease on a preoperative CT scan is predictive for anastomotic leakage after esophagectomy. Eur J Surg Oncol. 2018;44(5):587-93.

20. van der Werf LR, Busweiler LAD, van Sandick JW, van Berge Henegouwen MI, Wijnhoven BPL. Reporting national outcomes after esophagectomy and gastrectomy according to the Esophageal Complications Consensus Group (ECCG). Ann Surg. 2019.

21. Van Daele E, Van de Putte D, Ceelen W, Van Nieuwenhove Y, Pattyn P. Risk factors and consequences of anastomotic leakage after Ivor Lewis oesophagectomy $\dagger$. Interactive Cardiovasc Thorac Surg. 2016;22(1):32-7.

22. Filip B, Scarpa M, Cavallin F, Cagol M, et al. Postoperative outcome after oesophagectomy for cancer: nutritional status is the missing ring in the current prognostic scores. Eur J Surg Oncol. 2015;41(6):787-94.

23. Fjederholt KT, Okholm C, Svendsen LB, Achiam MP, Kirkegard J, Mortensen FV. Ketorolac and other NSAIDs increase the risk of anastomotic leakage after surgery for GEJ cancers: a cohort study of 557 patients. J Gastrointest Surg. 2018;22(4):587-94.

24. Fogh SE, Yu A, Kubicek GJ, et al. Do elderly patients experience increased perioperative or postoperative morbidity or mortality when given neoadjuvant chemoradiation before esophagectomy? Int J Radiat Oncol Biol Phys. 2011;80(5):1372-6.

25. Gao C, Xu G, Wang C, Wang D. Evaluation of preoperative risk factors and postoperative indicators for anastomotic leak of minimally invasive McKeown esophagectomy: a single-center retrospective analysis. J Cardiothorac Surg. 2019;14(1):46.

26. Goense L, van Rossum PSN, Weijs TJ, et al. Aortic calcification increases the risk of anastomotic leakage after Ivor-Lewis esophagectomy. Ann Thorac Surg. 2016;102(1):247-52.

27. Gooszen JAH, Goense L, Gisbertz SS, Ruurda JP, van Hillegersberg R, van Berge Henegouwen MI. Intrathoracic versus cervical anastomosis and predictors of anastomotic leakage after oesophagectomy for cancer. Br J Surg. 2018;105(5):552-60.

28. Hall BR, Flores LE, Parshall ZS, Shostrom VK, Are C, Reames $\mathrm{BN}$. Risk factors for anastomotic leak after esophagectomy for cancer: A NSQIP procedure-targeted analysis. J Surg Oncol. 2019;120(4):661-9.

29. Harustiak T, Pazdro A, Snajdauf M, Stolz A, Lischke R. Anastomotic leak and stricture after hand-sewn versus linear-stapled intrathoracic oesophagogastric anastomosis: single-centre analysis of 415 oesophagectomies. Eur $J$ Cardiothorac Surg. 2016;49(6):1650-9.

30. Harustiak T, Pazdro A, Snajdauf M, Stolz A, Lischke R. The Surgical Apgar Score in esophagectomy. J Thorac Cardiovasc Surg. 2015;150(4):806-12.

31. Kassis ES, Kosinski AS, Ross P Jr, Koppes KE, Donahue JM, Daniel VC. Predictors of anastomotic leak after esophagectomy: an analysis of the society of thoracic surgeons general thoracic database. Ann Thorac Surg. 2013;96(6):1919-26.

32. Kathiravetpillai N, Koeter M, van der Sangen MJ, et al. Delaying surgery after neoadjuvant chemoradiotherapy does not significantly influence postoperative morbidity or oncological outcome in patients with oesophageal adenocarcinoma. Eur J Surg Oncol. 2016;42(8):1183-90.

33. Klevebro F, Elliott JA, Slaman A, et al. Cardiorespiratory comorbidity and postoperative complications following esophagectomy: a European multicenter cohort study. Ann Surg Oncol. 2019;26(9):2864-73.

34. Koeter M, van der Sangen MJ, Hurkmans CW, Luyer MD, Rutten HJ, Nieuwenhuijzen GA. Radiation dose does not influence anastomotic complications in patients with esophageal cancer treated with neoadjuvant chemoradiation and transhiatal esophagectomy. Radiat Oncol. 2015;10:59.

35. Koyanagi K, Ozawa S, Oguma J, et al. Blood flow speed of the gastric conduit assessed by indocyanine green fluorescence: New predictive evaluation of anastomotic leakage after esophagectomy. Medicine. 2016;95(30):e4386.

36. Kruhlikava I, Kirkegard J, Mortensen FV, Kjaer DW. Impact of body mass index on complications and survival after surgery for esophageal and gastro-esophageal-junction cancer. Scand J Surg. 2017;106(4):305-10.

37. Markar SR, Low DE. Physiology, not chronology, dictates outcomes after esophagectomy for esophageal cancer: outcomes in patients 80 years and older. Ann Surg Oncol. 2013;20(3):1020-6.

38. McBee PJ, Walters RW, Nandipati KC. Obesity is associated with significantly more anastomotic leaks after minimally invasive esophagectomy: a NSQIP database study. Ann Surg Oncol. 2020;27(9):3208-17.

39. Miki Y, Toyokawa T, Kubo N, et al. C-reactive protein indicates early stage of postoperative infectious complications in patients following minimally invasive esophagectomy. World J Surg. 2017;41(3):796-803.

40. Mitzman B, Schipper PH, Edwards MA, Kim S, Ferguson MK. Complications after esophagectomy are associated with extremes of body mass index. Ann Thorac Surg. 2018;106(4):973-80.

41. Miyawaki Y, Sato H, Fujiwara N, Sugita H, et al. Evaluation of the Associations between Gastric Tube Preparation Methods and the Incidence of Cervical Anastomotic Leakage after Esophagectomy for Thoracic Esophageal Cancer. Vol 37. Switzerland (2020).

42. Murphy CC, Incalcaterra JR, Albright HW, Correa AM, Swisher SG, Hofstetter WL. Pretreatment patient comorbidity and tobacco use increase cost and risk of postoperative complications after esophagectomy at a high-volume cancer center. J Oncol Pract. 2013;9(5):233-9. 
43. Okamura A, Watanabe M, Mine S, Nishida K, Kurogochi T, Imamura Y. Spirometric lung age predicts postoperative pneumonia after esophagectomy. World J Surg. 2016;40(10):2412-8.

44. Rutegard M, Lagergren P, Johar A, Lagergren J. Time shift in early postoperative mortality after oesophagectomy for cancer. Ann Surg Oncol. 2015;22(9):3144-9.

45. Rutegard M, Lagergren P, Rouvelas I, Lagergren J. Intrathoracic anastomotic leakage and mortality after esophageal cancer resection: a population-based study. Ann Surg Oncol. 2012;19(1):99-103.

46. Saito T, Tanaka K, Ebihara Y, et al. Novel prognostic score of postoperative complications after transthoracic minimally invasive esophagectomy for esophageal cancer: a retrospective cohort study of 90 consecutive patients. Esophagus. 2019;16(2):155-61.

47. Salem AI, Thau MR, Strom TJ, et al. Effect of body mass index on operative outcome after robotic-assisted Ivor-Lewis esophagectomy: retrospective analysis of 129 cases at a single high-volume tertiary care center. Dis Esophag. 2017;30(1):1-7.

48. Sato S, Nakatani E, Higashizono K, Nagai E, et al. Size of the thoracic inlet predicts cervical anastomotic leak after retrosternal reconstruction after esophagectomy for esophageal cancer. Surgery. 2020;168(3):558-66

49. Scarpa M, Filip B, Cavallin F, et al. Esophagectomy in elderly patients: which is the best prognostic score? Dis Esophag. 2016;29(6):589-97.

50. Schlottmann F, Strassle PD, Nayyar A, Herbella FAM, Cairns BA, Patti MG. Postoperative outcomes of esophagectomy for cancer in elderly patients. J Surg Res. 2018;229:9-14.

51. Shichinohe T, Uemura S, Hirano S, Hosokawa M. Impact of preoperative skeletal muscle mass and nutritional status on shortand long-term outcomes after esophagectomy for esophageal cancer: a retrospective observational study: impact of psoas muscle mass and body mass on esophagectomy. Ann Surg Oncol. 2019;26(5):1301-10.

52. Takeuchi $\mathrm{H}$, Miyata $\mathrm{H}$, Gotoh $\mathrm{M}$, et al. A risk model for esophagectomy using data of 5354 patients included in a Japanese nationwide web-based database. Ann Surg. 2014;260(2):259-66.

53. van der Werf LR, Dikken JL, van der Willik EM, van Berge Henegouwen MI, Nieuwenhuijzen GAP, Wijnhoven BPL. Time interval between neoadjuvant chemoradiotherapy and surgery for oesophageal or junctional cancer: a nationwide study. Eur $J$ Cancer. 2018;91:76-85.

54. Zhao H, Liu G, Wei S, Liu H. Short- and long-term outcomes of minimally invasive esophagectomy in elderly patients with esophageal squamous cell carcinoma. J BUON. 2017;22(6):1540-6.

55. Sharma A, Deeb AP, Iannuzzi JC, Rickles AS, Monson JR, Fleming FJ. Tobacco smoking and postoperative outcomes after colorectal surgery. Ann Surg. 2013;258(2):296-300.

56. Scheingraber S, Dobbert D, Schmiedel P, Seliger E, Dralle H. Gender-specific differences in sex hormones and cytokines in patients undergoing major abdominal surgery. Surg Today. 2005;35(10):846-54.

57. Goense L, Meziani J, Ruurda JP, van Hillegersberg R. Impact of postoperative complications on outcomes after oesophagectomy for cancer. Br J Surg. 2019;106(1):111-9.

58. Li SJ, Wang ZQ, Li YJ, et al. Diabetes mellitus and risk of anastomotic leakage after esophagectomy: a systematic review and meta-analysis. Dis Esophag. 2017;30(6):1-12.

59. Ri M, Aikou S, Seto Y. Obesity as a surgical risk factor. Ann Gastroenterol Surg. 2018;2(1):13-21.

60. Siewert JR, Stein HJ, Feith M, Bruecher BL, Bartels H, Fink U. Histologic tumor type is an independent prognostic parameter in esophageal cancer: lessons from more than 1,000 consecutive resections at a single center in the Western world. Ann Surg. 2001;234(3):360-9.
61. Goense L, van Dijk WA, Govaert JA, van Rossum PS, Ruurda JP, van Hillegersberg R. Hospital costs of complications after esophagectomy for cancer. Eur $J$ Surg Oncol. 2017;43(4):696-702.

62. Huang ZD, Gu HY, Zhu J, et al. The application of enhanced recovery after surgery for upper gastrointestinal surgery: metaanalysis. BMC Surg. 2020;20(1):3.

63. Oppedal K, Møller AM, Pedersen B, Tønnesen H. Preoperative alcohol cessation prior to elective surgery. Cochrane Database Syst Rev. 2012(7):Cd008343.

64. Ljungqvist O, de Boer HD, Balfour A, et al. Opportunities and challenges for the next phase of Enhanced Recovery after surgery: a review. JAMA Surg. 2021.

65. Nevo Y, Arjah S, Katz A, et al. ERAS 2.0: Continued refinement of an established enhanced recovery protocol for esophagectomy. Ann Surg Oncol. 2021.

66. Ding H, Xu J, You J, Qin H, Ma H. Effects of enteral nutrition support combined with enhanced recovery after surgery on the nutritional status, immune function, and prognosis of patients with esophageal cancer after Ivor-Lewis operation. J Thorac Dis. 2020;12(12):7337-45.

67. Mukai A, Suehiro K, Watanabe R, et al. Impact of intraoperative goal-directed fluid therapy on major morbidity and mortality after transthoracic oesophagectomy: a multicentre, randomised controlled trial. Br J Anaesth. 2020;125(6):953-61.

68. Kataoka K, Takeuchi H, Mizusawa J, et al. Prognostic impact of postoperative morbidity after esophagectomy for esophageal cancer: exploratory analysis of JCOG9907. Ann Surg. 2017;265(6):1152-7.

69. Ogura M, Takeuchi $\mathrm{H}$, Kawakubo $\mathrm{H}$, et al. Clinical significance of CXCL-8/CXCR-2 network in esophageal squamous cell carcinoma. Surgery. 2013;154(3):512-20.

70. Bedenne L, Michel P, Bouché O, et al. Chemoradiation followed by surgery compared with chemoradiation alone in squamous cancer of the esophagus: FFCD 9102. J Clin Oncol. 2007;25(10):1160-8.

71. Stahl M, Stuschke M, Lehmann N, et al. Chemoradiation with and without surgery in patients with locally advanced squamous cell carcinoma of the esophagus. $J$ Clin Oncol. 2005;23(10):2310-7.

72. Bolger JC, Loughney L, Tully R, et al. Perioperative prehabilitation and rehabilitation in esophagogastric malignancies: a systematic review. Dis Esophagus. 2019;32(9).

73. Argudo N, Rodó-Pin A, Martínez-Llorens J, et al. Feasibility, tolerability, and effects of exercise-based prehabilitation after neoadjuvant therapy in esophagogastric cancer patients undergoing surgery: an interventional pilot study. Dis Esophagus. 2021;34(4).

74. Soeters P, Bozzetti F, Cynober L, Elia M, Shenkin A, Sobotka L. Meta-analysis is not enough: the critical role of pathophysiology in determining optimal care in clinical nutrition. Clin Nutr. 2016;35(3):748-57.

75. Steenhagen E, van Vulpen JK, van Hillegersberg R, May AM, Siersema PD. Nutrition in peri-operative esophageal cancer management. Expert Rev Gastroenterol Hepatol. 2017;11(7):663-72.

76. Weenk M, Koeneman M, van de Belt TH, Engelen L, van Goor $\mathrm{H}$, Bredie SJH. Wireless and continuous monitoring of vital signs in patients at the general ward. Resuscitation. 2019;136:47-53.

77. Downey C, Randell R, Brown J, Jayne DG. Continuous versus intermittent vital signs monitoring using a wearable, wireless patch in patients admitted to surgical wards: pilot cluster randomized controlled trial. J Med Internet Res. 2018;20(12):e10802.

78. Beck N, van Bommel AC, Eddes EH, van Leersum NJ, Tollenaar RA, Wouters MW. The Dutch Institute for Clinical Auditing: 
achieving Codman's dream on a nationwide basis. Ann Surg. 2020;271(4):627-31.

79. Busweiler LA, Wijnhoven BP, van Berge Henegouwen MI, et al. Early outcomes from the Dutch Upper Gastrointestinal Cancer Audit. Br J Surg. 2016;103(13):1855-63.

80. Riley RD, Hayden JA, Steyerberg EW, et al. Prognosis Research Strategy (PROGRESS) 2: prognostic factor research. PLoS Med. 2013;10(2):e1001380.

81. Steyerberg EW, Kievit J, de Mol Van Otterloo JC, van Bockel JH, Eijkemans MJ, Habbema JD. Perioperative mortality of elective abdominal aortic aneurysm surgery. A clinical prediction rule based on literature and individual patient data. Arch Intern Med. 1995;155(18):1998-2004.

Publisher's Note Springer Nature remains neutral with regard to jurisdictional claims in published maps and institutional affiliations. 